\title{
Evidence points to the existence of cancer stem cells
}

Currently, in solid tumours there are two models for tumour proliferation. First, the classic theory in which all cancer cells have proliferative potential and can replicate and contribute to the expansion of the tumour. Second, the cancer stem cell (CSC) hypothesis suggests that there is a hierarchy of cell proliferation with a distinct population of cancer cells at the top of the pyramid. Now, two studies published in Nature and one in Science have provided evidence for the presence of CSCs in solid tumours for the first time in models of unperturbed tumour growth.

The first study, led by Gregory Driessens, Cédric Blanpain and Ben Simons, used a chemically induced carcinogenesis mouse model of early stage squamous-skin tumours. Driessens explains that "so far, the existence of cancer stem cells in different solid tumour models has always relied on grafting experiments that show what a cell can do, but not necessarily what it really does in its natural environment."
Their clonal analysis strategy revealed that most tumour cells in benign papilloma have only a limited proliferative potential. A fraction of tumour cells persist long term with the capacity to give rise to progeny that go on to make up a large proportion of the tumour. Driessens notes: "for the first time, we show the existence of CSCs in an unperturbed tumour. Our finding confirms that clonal analysis can be used to demonstrate the presence of such cells in solid tumours and that CSCs really exist as was suggested, but not formally proven, by grafting experiments."

The second study, led by Luis Parada, used a genetically engineered mouse model with $100 \%$ prevalence of glioblastoma, which has been shown to arise from normal stem cells that acquire mutations. These mice had an additional transgene introduced and were treated with the chemotherapy agent temozolomide, and the tumour cell population observed. They identified a subset of, usually quiescent, endogenous tumour cells that are capable of repopulating the tumour. Parada points out: "this demonstrates a tumour cell hierarchy in which a relatively quiescent CSC (that serendipitously expresses our transgene) gives rise to actively dividing cells. Better understanding of the CSCs will be critical for re-evaluation of existing therapies and development of new ones."

The final study, led by Hans Clevers, also used lineage tracing to demonstrate the presence of CSCs, but in mouse models of primary intestinal adenomas. Taken together, these three studies add considerable weight to the theory of CSCs and may drive future clinical research.

\section{Rebecca Kirk}

Original articles Driessens, G. et al. Defining the mode of
tumour growth by clonal analysis. Nature doi:10.1038/
nature11344 | Chen, J. et al. A restricted cell population
propagates glioblastoma growth after chemotherapy. Nature
doi:10.1038/nature11287 | Schepers, A. G. et al. Lineage
tracing reveals Lgr5+ stem cell activity in mouse intestinal
adenomas. Science doi:10.1126/science.1224676

